\title{
Monte Carlo comparison of estimation methods for the two-parameter lognormal distribution
}

\author{
Ivana Pobočíková $^{1}$, Zuzana Sedliačková1, Mária Michalková ${ }^{1,}$, and Lenka Kucharikováa \\ ${ }^{1}$ Department of Applied Mathematics, Faculty of Mechanical Engineering, University of Žilina, \\ Univerzitná 8215/1, 01026 Žilina, Slovakia \\ ${ }^{2}$ Department of Materials Engineering, Faculty of Mechanical Engineering, University of Žilina, \\ Univerzitná 8215/1, 01026 Žilina, Slovakia
}

\begin{abstract}
In the paper we compare performance of estimation methods for the two-parameter lognormal distribution via the Monte Carlo simulation. The comparison of performances is made with respect to their biases, variances, root mean square error. The methods are applied on real data set representing experimentally obtained values of ultimate tensile strength of material.
\end{abstract}

Keywords: lognormal distribution, maximum likelihood method, method of moments, Finney method, Monte Carlo simulation, ultimate tensile strength

\section{Introduction}

It is well known that the lognormal distribution is frequently used in areas where the data tend to be right skewed, such as physics, economics, biostatistics, survival analysis, wireless communication, quality control, reliability analysis, material properties, fatigue life of materials, strength of some materials, etc. Various applications of the lognormal distribution are described in [1-4].

The aim of this paper is to present methods for estimation of the parameters of lognormal distribution. Here, three estimation methods are used, namely the maximum likelihood method (MLM), the method of moments (MOM) and the Finney method (UMVUE). The performance of these methods is compared using the Monte Carlo simulation study. The comparison of the estimation methods is based on the biases and the root mean square error (RMSE). For the purpose of simulation, mathematical software MATLAB R2017b is used.

The rest of the paper is organized as follows: in Section 2 definition and characteristics of the lognormal distribution are summarized. In Section 3 the estimation methods are briefly introduced. In Section 4 we present results of the Monte Carlo simulation study. The real data application is provided in Section 5 and finally conclusion appears in Section 6.

\footnotetext{
*Corresponding author: maria.michalkova@fstroj.uniza.sk

Reviewers: Mariana Pajtašová, Leszek Radziszewski
} 


\section{Lognormal distribution}

The random variable $X$ is said to have the 2-parameter lognormal distribution $L N\left(\mu, \sigma^{2}\right)$ with parameters $\mu$ and $\sigma^{2}$ if its probability density function (PDF) $f(x)$ and the cumulative distribution function (CDF) $F(x)$ are given as

$$
\begin{gathered}
f(x)=\frac{1}{x \sigma \sqrt{2 \pi}} \exp \left(-\frac{1}{2}\left(\frac{\ln x-\mu}{\sigma}\right)^{2}\right) \\
F(x)=\Phi\left(\frac{\ln x-\mu}{\sigma}\right)
\end{gathered}
$$

respectively, for $x>0, \mu \in R, \sigma>0$. Here $\Phi(x)$ is the cumulative distribution function of standard normal distribution $N(0,1)$.

The $r$-th moment, $r=1,2,3, \ldots$, of the lognormal distribution is defined as

$$
E\left(X^{r}\right)=\exp \left(r \mu+r^{2} \frac{\sigma^{2}}{2}\right)
$$

The mean $E(X)$ and the variance $\operatorname{Var}(X)$ are given by

$$
\begin{gathered}
E(X)=\exp \left(\mu+\frac{\sigma^{2}}{2}\right) \\
\operatorname{Var}(X)=\exp \left(2 \mu+\sigma^{2}\right)\left[\exp \left(\sigma^{2}\right)-1\right]
\end{gathered}
$$

The lognormal distribution is a right skewed distribution with a long tail. Figure 1 illustrates the PDF curves for different parameter values. 


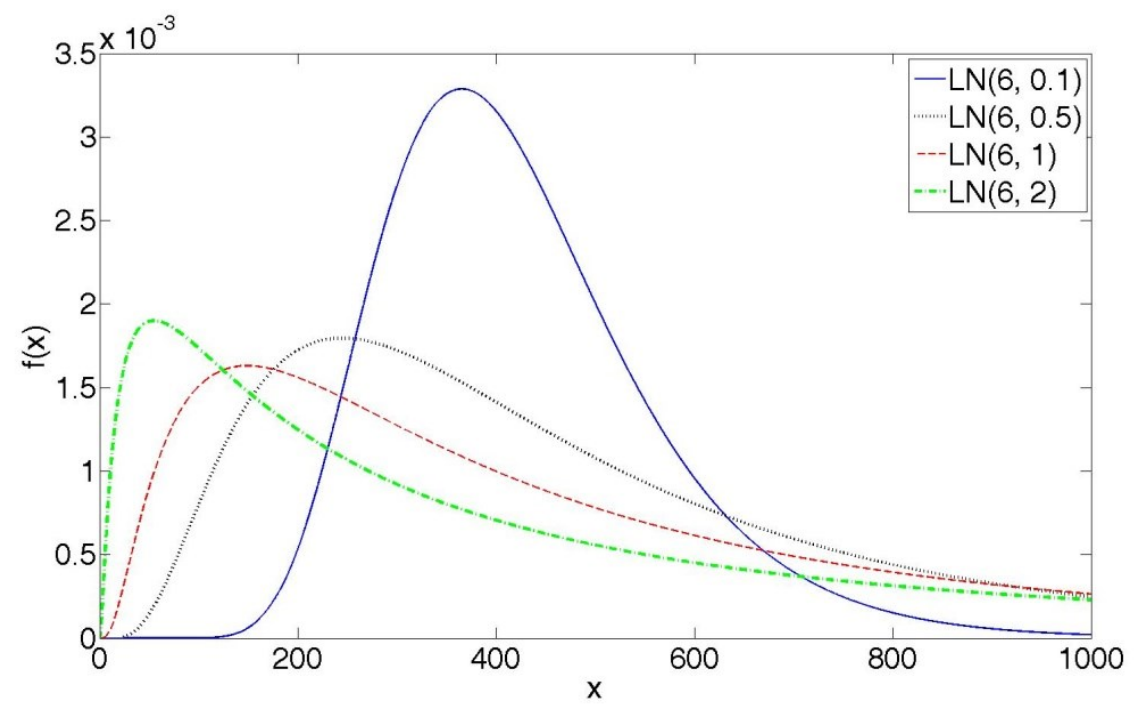

Fig. 1. PDF of the lognormal distribution for different parameter values

There is a close relationship between the lognormal and the normal distribution. If $X$ is the random variable that has lognormal distribution $L N\left(\mu, \sigma^{2}\right)$, then the random variable $Y=\ln X$ has normal distribution $N\left(\mu, \sigma^{2}\right)$.

\section{Methods of estimation}

In this section, we briefly describe the methods for estimation of parameters $\mu$ and $\sigma^{2}$ of the lognormal distribution $L N\left(\mu, \sigma^{2}\right)$.

\subsection{Maximum likelihood method}

The maximum likelihood method (MLM) is one of widely used parameter estimation methods. It is defined as follows: Let $x_{1}, x_{2}, \ldots, x_{n}$ be a realization of a random sample of size $\mathrm{n}$ from the lognormal distribution $L N\left(\mu, \sigma^{2}\right)$. The likelihood function is given by

$$
L\left(x_{1}, x_{2}, \ldots, x_{n}, \mu, \sigma^{2}\right)=\left(2 \pi \sigma^{2}\right)^{-n / 2} \exp \left(-\frac{1}{2 \sigma^{2}} \sum_{i=1}^{n}\left(\ln x_{i}-\mu\right)^{2}\right) \prod_{i=1}^{n} x_{i} .
$$

Taking the natural logarithm of the likelihood function (6) we obtain

$$
\ln L\left(x_{1}, x_{2}, \ldots, x_{n}, \mu, \sigma^{2}\right)=-\frac{n}{2} \ln \left(2 \pi \sigma^{2}\right)-\frac{1}{2 \sigma^{2}} \sum_{i=1}^{n}\left(\ln x_{i}-\mu\right)^{2}-\sum_{i=1}^{n} \ln x_{i} .
$$

The MLM estimates $\hat{\mu}$ and $\hat{\sigma}^{2}$ of parameters $\mu$ and $\sigma^{2}$ which maximize (7) are given by

$$
\hat{\mu}=\frac{1}{n} \sum_{i=1}^{n} \ln x_{i}
$$




$$
\hat{\sigma}^{2}=\frac{1}{n} \sum_{i=1}^{n}\left(\ln x_{i}-\frac{1}{n} \sum_{i=1}^{n} \ln x_{i}\right)^{2}
$$

Estimates $\hat{M}$ and $\hat{V}$ of the mean $E(X)$ and the variance $\operatorname{Var}(X)$, respectively, are given by

$$
\begin{gathered}
\hat{M}=\exp \left(\hat{\mu}+\frac{\hat{\sigma}^{2}}{2}\right) \\
\hat{V}=\exp \left(2 \hat{\mu}+\hat{\sigma}^{2}\right)\left[\exp \left(\hat{\sigma}^{2}\right)-1\right]
\end{gathered}
$$

where $\hat{\mu}$ and $\hat{\sigma}^{2}$ are given by equations (8), (9).

\subsection{Method of moments}

The estimates, based on the method of moments (MOM), are obtained by equating the distribution moments to corresponding sample moments. The MOM estimates $\hat{\mu}$ and $\hat{\sigma}^{2}$ of the parameters $\mu$ and $\sigma^{2}$ can be easily calculated using following equations

$$
\begin{aligned}
& \hat{\mu}=\ln \left(\frac{1}{n} \sum_{i=1}^{n} x_{i}\right)-\frac{1}{2} \ln \frac{\frac{1}{n} \sum_{i=1}^{n} x_{i}^{2}}{\left(\frac{1}{n} \sum_{i=1}^{n} x_{i}\right)^{2}} \\
& \hat{\sigma}^{2}=\ln \left(\frac{1}{n} \sum_{i=1}^{n} x_{i}^{2}\right)-2 \ln \left(\frac{1}{n} \sum_{i=1}^{n} x_{i}\right)
\end{aligned}
$$

Estimates $\hat{M}$ and $\hat{V}$ of the mean $E(X)$ and the variance $\operatorname{Var}(X)$, respectively, are given by

$$
\begin{gathered}
\hat{M}=\exp \left(\hat{\mu}+\frac{\hat{\sigma}^{2}}{2}\right) \\
\hat{V}=\exp \left(2 \hat{\mu}+\hat{\sigma}^{2}\right)\left[\exp \left(\hat{\sigma}^{2}\right)-1\right]
\end{gathered}
$$

where $\hat{\mu}$ and $\hat{\sigma}^{2}$ are given by equations (12), (13).

\subsection{Finney method}

Finney [5] defined the series 


$$
\begin{aligned}
g(t)= & +t \frac{n-1}{n}+t^{2} \frac{(n-1)^{3}}{n^{2}(n+1) 2 !}+t^{3} \frac{(n-1)^{5}}{n^{3}(n+1)(n+3) 3 !}+ \\
& +t^{4} \frac{(n-1)^{7}}{n^{4}(n+1)(n+3)(n+5) 4 !}+\cdots
\end{aligned}
$$

and obtained the uniformly minimum variance unbiased estimators (UMVUE) $\hat{M}$ of the mean $E(X)$ and $\hat{V}$ of the variance $\operatorname{Var}(X)$ as

$$
\begin{gathered}
\hat{M}=\exp (\bar{Y}) g\left(\frac{s^{2}}{2}\right) \\
\hat{V}=\exp (2 \bar{Y})\left[g\left(2 s^{2}\right)-g\left(\frac{n-2}{n-1} s^{2}\right)\right]
\end{gathered}
$$

where $Y_{i}=\ln x_{i}, i=1,2, \ldots, n ; \bar{Y}=\frac{1}{n} \sum_{i=1}^{n} Y_{i}$ and $s^{2}=\frac{1}{n-1} \sum_{i=1}^{n}\left(Y_{i}-\bar{Y}\right)^{2}$.

Depending on $n$ and $s^{2}, 6$ to 10 terms must be evaluated to achieve stability. Thus, the estimates $\hat{\mu}$ and $\hat{\sigma}^{2}$ of the parameters $\mu$ and $\sigma^{2}$ are

$$
\begin{gathered}
\hat{\mu}=2 \ln \hat{M}-\frac{1}{2} \ln \left(\hat{M}^{2}+\hat{V}\right), \\
\hat{\sigma}^{2}=\ln \left(\hat{M}^{2}+\hat{V}\right)-2 \ln \hat{M} .
\end{gathered}
$$

\section{Simulation study}

Numerical simulations provide many benefits when modelling in science and technology. They are used for checking various scenarios, for comparison of different approaches or methods, or in first steps of designing devices (see, for example, [6-9]).

We use the Monte Carlo simulation for comparison of the performances of the chosen estimation methods for the lognormal distribution parameters. In simulation we consider $\mu=6$ with $\sigma^{2}=0.1,0.5,1,2$. The sample sizes are $n=10,20,50,100$ in order to cover small and medium sample sizes. For each combination of parameter values and sample sizes, $N=5000$ random samples from the lognormal distribution are simulated by the Monte Carlo

simulation. For each method discussed, we compute the sample means $\bar{\mu}$ and $\overline{\sigma^{2}}$, and the sample variances $s_{\mu}^{2}$ and $s_{\sigma^{2}}^{2}$ as

$$
\bar{\mu}=\frac{1}{N} \sum_{i=1}^{N} \hat{\mu}_{i}, \quad \overline{\sigma^{2}}=\frac{1}{N} \sum_{i=1}^{N} \hat{\sigma}_{i}^{2}
$$




$$
s_{\mu}^{2}=\frac{1}{N-1} \sum_{i=1}^{N}\left(\hat{\mu}_{i}-\bar{\mu}\right)^{2}, \quad s_{\sigma^{2}}^{2}=\frac{1}{N-1} \sum_{i=1}^{N}\left(\hat{\sigma}_{i}^{2}-\overline{\sigma^{2}}\right)^{2},
$$

respectively.

For comparing the performances of the methods we consider the bias given by

$$
\operatorname{bias}(\hat{\mu})=\bar{\mu}-\mu, \quad \operatorname{bias}\left(\hat{\sigma}^{2}\right)=\overline{\sigma^{2}}-\sigma^{2},
$$

respectively, and the sample root mean square error (RMSE) given by

$$
\operatorname{RMSE}(\hat{\mu})=\sqrt{\frac{1}{N} \sum_{i=1}^{N}\left(\hat{\mu}_{i}-\mu\right)^{2}}, \quad \operatorname{RMSE}\left(\hat{\sigma}^{2}\right)=\sqrt{\frac{1}{N} \sum_{i=1}^{N}\left(\hat{\sigma}_{i}^{2}-\sigma^{2}\right)^{2}}
$$

respectively.

Ideal value of $R M S E$ is close to zero. The methods with smaller RMSE are preferred. When RMSE values of two methods are close to each other, the method with less bias is preferred.

Table 1. Simulation results for $\mu=6$ and $\sigma^{2}=0.1$

\begin{tabular}{|l|c|c|c|c|c|c|c|c|}
\hline Method & $\bar{\mu}$ & $\overline{\sigma^{2}}$ & $s_{\mu}^{2}$ & $s_{\sigma^{2}}^{2}$ & $\operatorname{bias}(\hat{\mu})$ & $\operatorname{bias}\left(\hat{\sigma}^{2}\right)$ & $R M S E(\hat{\mu})$ & $R M S E\left(\hat{\sigma}^{2}\right)$ \\
\hline MLM & 5.9976 & 0.0906 & 0.0101 & 0.0018 & -0.0024 & -0.0094 & 0.1005 & 0.0434 \\
MOM & 5.9991 & 0.0870 & 0.0101 & 0.0019 & -0.0009 & -0.0130 & 0.1005 & 0.0454 \\
UMVUE & 5.9942 & 0.0968 & 0.0101 & 0.0019 & -0.0058 & -0.0032 & 0.1006 & 0.0437 \\
\hline \multicolumn{10}{|c|}{$n=20$} \\
\hline MLM & 5.9994 & 0.0949 & 0.0049 & 0.0009 & -0.0006 & -0.0052 & 0.0698 & 0.0310 \\
MOM & 6.0001 & 0.0932 & 0.0049 & 0.0011 & 0.0001 & -0.0068 & 0.0700 & 0.0340 \\
UMVUE & 5.9977 & 0.0979 & 0.0049 & 0.0010 & -0.0023 & -0.0021 & 0.0698 & 0.0310 \\
\hline \multicolumn{10}{|c|}{$n=50$} \\
\hline MLM & 6.0000 & 0.0973 & 0.0020 & 0.0004 & -0.00001 & -0.0027 & 0.0447 & 0.0194 \\
MOM & 6.0003 & 0.0965 & 0.0020 & 0.0005 & 0.0003 & -0.0035 & 0.0449 & 0.0219 \\
UMVUE & 5.9993 & 0.0985 & 0.0020 & 0.0004 & -0.0007 & -0.0015 & 0.0447 & 0.0194 \\
\hline \multicolumn{10}{|c|}{$n=100$} \\
\hline MLM & 5.9991 & 0.0989 & 0.0010 & 0.0002 & -0.0009 & -0.0011 & 0.0319 & 0.0143 \\
MOM & 5.9993 & 0.0984 & 0.0010 & 0.0003 & -0.0007 & -0.0016 & 0.0321 & 0.0164 \\
UMVUE & 5.9988 & 0.0995 & 0.0010 & 0.0002 & -0.0012 & -0.0005 & 0.0319 & 0.0142 \\
\hline
\end{tabular}

Table 2. Simulation results for $\mu=6$ and $\sigma^{2}=0.5$

\begin{tabular}{|c|c|c|c|c|c|c|c|c|}
\hline Method & $\bar{\mu}$ & $\overline{\sigma^{2}}$ & $s_{\mu}^{2}$ & $s_{\sigma^{2}}^{2}$ & $\operatorname{bias}(\hat{\mu})$ & $\operatorname{bias}\left(\hat{\sigma}^{2}\right)$ & $\operatorname{RMSE}(\hat{\mu})$ & $\operatorname{RMSE}\left(\hat{\sigma}^{2}\right)$ \\
\hline \multicolumn{1}{|c|}{$n=20$} \\
\hline MLM & 5.9970 & 0.4468 & 0.0500 & 0.0433 & -0.0030 & -0.0532 & 0.2236 & 0.2148 \\
MOM & 6.0314 & 0.3653 & 0.0519 & 0.0342 & 0.0314 & -0.1347 & 0.2299 & 0.2288 \\
UMVUE & 6.0056 & 0.4191 & 0.0503 & 0.0281 & 0.0056 & -0.0809 & 0.2243 & 0.1862 \\
\hline
\end{tabular}




\begin{tabular}{|c|c|c|c|c|c|c|c|c|}
\hline Method & $\bar{\mu}$ & $\overline{\sigma^{2}}$ & $s_{\mu}^{2}$ & $s_{\sigma^{2}}^{2}$ & bias $(\hat{\mu})$ & bias $\left(\hat{\sigma}^{2}\right)$ & $R M S E(\hat{\mu})$ & $R M S E\left(\hat{\sigma}^{2}\right)$ \\
\hline MLM & 6.0007 & 0.4730 & 0.0249 & 0.0236 & 0.0007 & -0.0270 & 0.1577 & 0.1560 \\
MOM & 6.0240 & 0.4206 & 0.0266 & 0.0303 & 0.0240 & -0.0794 & 0.1619 & 0.1914 \\
UMVUE & 6.0072 & 0.4543 & 0.0249 & 0.0183 & 0.0072 & -0.0457 & 0.1579 & 0.1427 \\
\hline \multicolumn{7}{|c|}{$n=50$} \\
\hline MLM & 5.9974 & 0.4881 & 0.0100 & 0.0010 & -0.0026 & -0.0119 & 0.0997 & 0.0996 \\
MOM & 6.0100 & 0.4600 & 0.0115 & 0.0192 & 0.0101 & -0.0400 & 0.1078 & 0.1442 \\
UMVUE & 6.0001 & 0.4790 & 0.0099 & 0.0087 & 0.0008 & -0.0210 & 0.0997 & 0.0956 \\
\hline \multicolumn{7}{|c|}{$n=100$} & & \\
\hline \\
MLM
\end{tabular}

Table 3. Simulation results for $\mu=6$ and $\sigma^{2}=1$.

\begin{tabular}{|c|c|c|c|c|c|c|c|c|}
\hline Method & $\bar{\mu}$ & $\overline{\sigma^{2}}$ & $s_{\mu}^{2}$ & $s_{\sigma^{2}}^{2}$ & $\operatorname{bias}(\hat{\mu})$ & $\operatorname{bias}\left(\hat{\sigma}^{2}\right)$ & $R M S E(\hat{\mu})$ & $R M S E\left(\hat{\sigma}^{2}\right)$ \\
\hline \multicolumn{9}{|c|}{$n=10$} \\
\hline MLM & 6.0041 & 0.9039 & 0.0971 & 0.1785 & 0.0041 & -0.0961 & 0.3116 & 0.4332 \\
\hline MOM & 6.1211 & 0.6256 & 0.1085 & 0.0879 & 0.1211 & -0.3744 & 0.3510 & 0.4776 \\
\hline UMVUE & 6.0649 & 0.7420 & 0.1021 & 0.0706 & 0.0649 & -0.2580 & 0.3261 & 0.3703 \\
\hline \multicolumn{9}{|c|}{$n=20$} \\
\hline MLM & 5.9980 & 0.9577 & 0.0509 & 0.0962 & -0.0020 & -0.0423 & 0.2256 & 0.3129 \\
\hline MOM & 6.0934 & 0.7357 & 0.0575 & 0.0893 & 0.0933 & -0.2643 & 0.2574 & 0.3989 \\
\hline UMVUE & 6.0431 & 0.8450 & 0.0523 & 0.0523 & 0.0431 & -0.1550 & 0.2328 & 0.2763 \\
\hline \multicolumn{9}{|c|}{$n=50$} \\
\hline MLM & 5.9993 & 0.9811 & 0.0198 & 0.0387 & -0.0007 & -0.0189 & 0.1407 & 0.1977 \\
\hline MOM & 6.0621 & 0.8391 & 0.0258 & 0.0778 & 0.0621 & -0.1609 & 0.1721 & 0.3220 \\
\hline UMVUE & 6.0254 & 0.9193 & 0.0200 & 0.0273 & 0.0254 & -0.0807 & 0.1436 & 0.1839 \\
\hline \multicolumn{9}{|c|}{$n=100$} \\
\hline MLM & 5.9979 & 0.9909 & 0.0097 & 0.0199 & -0.0021 & -0.0091 & 0.0985 & 0.1415 \\
\hline MOM & 6.0384 & 0.9046 & 0.0160 & 0.0648 & 0.0384 & -0.0954 & 0.1320 & 0.2718 \\
\hline UMVUE & 6.0157 & 0.9502 & 0.0098 & 0.0155 & 0.0157 & -0.0498 & 0.1001 & 0.1339 \\
\hline
\end{tabular}

Table 4. Simulation results for $\mu=6$ and $\sigma^{2}=2$.

\begin{tabular}{|c|c|c|c|c|c|c|c|c|}
\hline Method & $\bar{\mu}$ & $\overline{\sigma^{2}}$ & $s_{\mu}^{2}$ & $s_{\sigma^{2}}^{2}$ & $\operatorname{bias}(\hat{\mu})$ & $\operatorname{bias}\left(\hat{\sigma}^{2}\right)$ & $\operatorname{RMSE}(\hat{\mu})$ & $\operatorname{RMSE}\left(\hat{\sigma}^{2}\right)$ \\
\hline \multicolumn{1}{|c|}{$n=10$} \\
\hline MLM & 6.0048 & 1.7730 & 0.1960 & 0.6967 & 0.0048 & -0.2270 & 0.4427 & 0.8649 \\
MOM & 6.3400 & 0.9261 & 0.2517 & 0.1522 & 0.3400 & -1.0739 & 0.6060 & 1.1426 \\
UMVUE & 6.2310 & 1.1795 & 0.2314 & 0.1230 & 0.2310 & -0.8205 & 0.5335 & 0.8923 \\
\hline
\end{tabular}




\begin{tabular}{|c|c|c|c|c|c|c|c|c|}
\hline \multicolumn{9}{|c|}{$n=20$} \\
\hline MLM & 5.9988 & 1.8965 & 0.0989 & 0.3742 & -0.0012 & -0.1035 & 0.3144 & 0.6204 \\
\hline MOM & 6.3120 & 1.1492 & 0.1296 & 0.1856 & 0.3120 & -0.8508 & 0.4764 & 0.9537 \\
\hline UMVUE & 6.1979 & 1.4138 & 0.1166 & 0.0923 & 0.1979 & -0.5862 & 0.3946 & 0.6603 \\
\hline \multicolumn{9}{|c|}{$n=50$} \\
\hline MLM & 5.9983 & 1.9516 & 0.0413 & 0.1523 & -0.0017 & -0.0484 & 0.2031 & 0.3932 \\
\hline MOM & 6.2466 & 1.3891 & 0.0580 & 0.2000 & 0.2466 & -0.6109 & 0.3446 & 0.7570 \\
\hline UMVUE & 6.1562 & 1.5981 & 0.0479 & 0.0483 & 0.1562 & -0.4019 & 0.2688 & 0.4581 \\
\hline \multicolumn{9}{|c|}{$n=100$} \\
\hline MLM & 5.9960 & 1.9787 & 0.0199 & 0.0804 & -0.0040 & -0.0213 & 0.1411 & 0.2844 \\
\hline MOM & 6.1950 & 1.5497 & 0.0358 & 0.2065 & 0.1950 & -0.4503 & 0.2717 & 0.6397 \\
\hline UMVUE & 6.1367 & 1.6769 & 0.0233 & 0.0273 & 0.1367 & -0.3231 & 0.2048 & 0.3629 \\
\hline
\end{tabular}

The results of the Monte Carlo simulation are presented in Table 1-4. From the results, the following can be observed, regards to RMSE and bias:

- RMSE of parameter $\mu$ : MLM achieves the smallest RMSE in all considered cases. In case $\mu=6, \sigma^{2}=0.1$, the performances of MLM and UMVUE are on the same level.

- RMSE of parameter $\sigma^{2}$ : For cases $\mu=6 ; \sigma^{2}=0.5$ and $\mu=6 ; \sigma^{2}=1$, UMVUE outperforms the other two methods. MLM has the smallest RMSE for $\mu=6 ; \sigma^{2}=2$. For $\mu=6 ; \sigma^{2}=0.1$, MLM and UMVUE perform similarly well.

- Furthermore, it can be observed that MOM has the highest values of RMSE for both estimated parameters. Hence, its performance is the worst in the terms of RMSE from the considered methods.

- Bias of parameter $\mu$ : MLM proves to be the best estimation method, with UMVUE as second best, except the case $\mu=6 ; \sigma^{2}=0.1$ when MOM achieves the smallest bias.

- Bias of parameter $\sigma^{2}$ : Similarly, MLM outperforms the other two methods in majority of cases. For $\mu=6 ; \sigma^{2}=0.1$, UMVUE performs the best.

- It can be seen that in all considered cases each method has negative bias for parameter $\sigma^{2}$.

\section{Real data example}

Engineering products are often subject to loads when they are used in specific applications. The mechanical properties of metals determine the range of applicability and establish the expected service life. Additionally, mechanical properties provide knowledge how materials deform (elongate, compress, twist), or break as a function of applied load, time, temperature, and other conditions. The most common mechanical properties, useful for industry, are ultimate tensile strength, ductility, hardness, impact resistance, and fracture toughness. Characteristics that indicate the elastic or inelastic behaviour of a material under pressure from tensile tests are ultimate tensile strength, yield stress, ductility, toughness and elongation. The maximum stress the material can withstand before fracture is known as ultimate tensile strength (UTS). The UTS represents maximal stress (load) which material withstands without fracture, to original cross section area before testing. Chemical composition, microstructure of material and others influence mechanical properties of material (see, e.g. $[10,11])$.

In this section we apply estimation methods, described in Section 3, to find estimates of parameters of the lognormal distribution $L N\left(\mu, \sigma^{2}\right)$ for data set representing UTS. 
As a testing material, common steel 1.0036 was used. The tensile tests were conducted on shredder machine ZDM 30. There were used round specimens with specified dimension according standard STN EN ISO 6892-1. The specimen was axially loaded; the stress (load) was being increased at a uniform rate until the fracture of the specimen. Stress and elongation were recorded during test.

The following data set represents the UTS (in MPa) from 20 tests of experimental material.

$$
\begin{array}{llllllllll}
448.1 & 449.4 & 450.8 & 445.6 & 457.4 & 452.1 & 448.1 & 444.1 & 450.8 & 456.1 \\
458.7 & 456.1 & 448.1 & 452.1 & 450.8 & 442.8 & 449.4 & 445.5 & 450.8 & 452.1
\end{array}
$$

Table 5 gives the descriptive statistics for the tensile strength data. The value of skewness indicates that the empirical distribution is skewed to the right.

Table 5. Descriptive statistics for the data set

\begin{tabular}{|c|c|c|c|c|c|c|c|c|}
\hline Min & Max & Mean & Variance & Lower Quantile & Median & Upper Quantile & Skewness & Kurtosis \\
\hline 442.8 & 458.7 & 450.445 & 18.653 & 448.1 & 450.8 & 452.1 & 0.215 & -0.358 \\
\hline
\end{tabular}

First, we compute the estimates of the lognormal parameters using methods described in Section 3. For comparison of the estimation methods, the Kolmogorov-Smirnov test is applied. We test the hypothesis:

$H_{0}$ : the data follow the specified distribution with $\operatorname{CDF} F(x)$,

$H_{A}$ : the data do not follow the specified distribution with $\mathrm{CDF} F(x)$.

The Kolmogorov-Smirnov test statistics is computed using

$$
D_{n}=\max _{1 \leq i \leq n}\left\{\left|\frac{i}{n}-F\left(x_{(i)}\right)\right|,\left|F\left(x_{(i)}\right)-\frac{i-1}{n}\right|\right\} .
$$

When $D_{n}>D_{n}(\alpha)$, we can reject the null hypothesis $H_{0}$ at the significance level $\alpha$ where $D_{n}(\alpha)$ is the critical value of the Kolmogorov-Smirnov test.

We also apply these model selection criteria:

- the Akaike's information criterion (AIC)[12]

$$
A I C=-2 \ln L+2 k,
$$

- the corrected Akaike's information criterion (AICC)

$$
A I C C=A I C+\frac{2 k(k+1)}{n-k+1}
$$

- the Bayesian information criterion (BIC) [13]

$$
B I C=-2 \ln L+k \ln (n)
$$

where $\ln L=\ln L\left(x_{1}, x_{2}, \ldots, x_{n}, \hat{\mu}, \hat{\sigma}^{2}\right)$ denotes the maximized value of the loglikelihood function, $\hat{\mu}$ and $\hat{\sigma}^{2}$ are the estimates of the parameters $\mu$ and $\sigma^{2}$, respectively, $k$ is number of the parameters to be estimated and $n$ is number of the observed data. For deciding, we consider the following rule: the smaller the values of $A I C, A I C C, B I C$, the better the fit to the data.

Table 6 presents the estimates of the parameters and the model selection criteria for the considered estimation methods. The critical value of the Kolmogorov-Smirnov test is 
$D_{20}(0.05)=0.294$. Based on the Kolmogorov-Smirnov tests we cannot reject the null hypothesis that the data follow the lognormal distribution.

Values in Table 6 indicate that all three methods provide similar results. The UMVUE is only slightly worse than the MLM and MOM.

Figure 2 shows the fitted PDF versus the histogram of the tensile strength for all three estimation methods.

Table 6. The estimates of the parameters, mean, variance and model selection criteria

\begin{tabular}{|c|c|c|c|c|c|c|c|c|}
\hline \multirow{2}{*}{ Method } & \multicolumn{2}{|c|}{ Parameter estimates } & \multicolumn{2}{|c|}{$\begin{array}{c}\text { Mean and variance } \\
\text { estimates }\end{array}$} & \multicolumn{4}{|c|}{ Statistical test } \\
\cline { 2 - 9 } & $\hat{\mu}$ & $\hat{\sigma}^{2}$ & $\hat{M}$ & $\hat{V}$ & KS & AIC & AICC & BIC \\
\hline MLM & 6.110192 & $8.72 \cdot 10^{-5}$ & 450.445 & 17.6934 & 0.1456 & 118.2221 & 118.8537 & 120.2136 \\
\hline MOM & 6.110258 & $8.74 \cdot 10^{-5}$ & 450.445 & 17.7255 & 0.1457 & 118.8638 & 118.8538 & 120.2136 \\
\hline UMVUE & 6.110190 & $9.18 \cdot 10^{-5}$ & 450.445 & 18.6275 & 0.1492 & 118.2480 & 118.8796 & 120.2395 \\
\hline
\end{tabular}

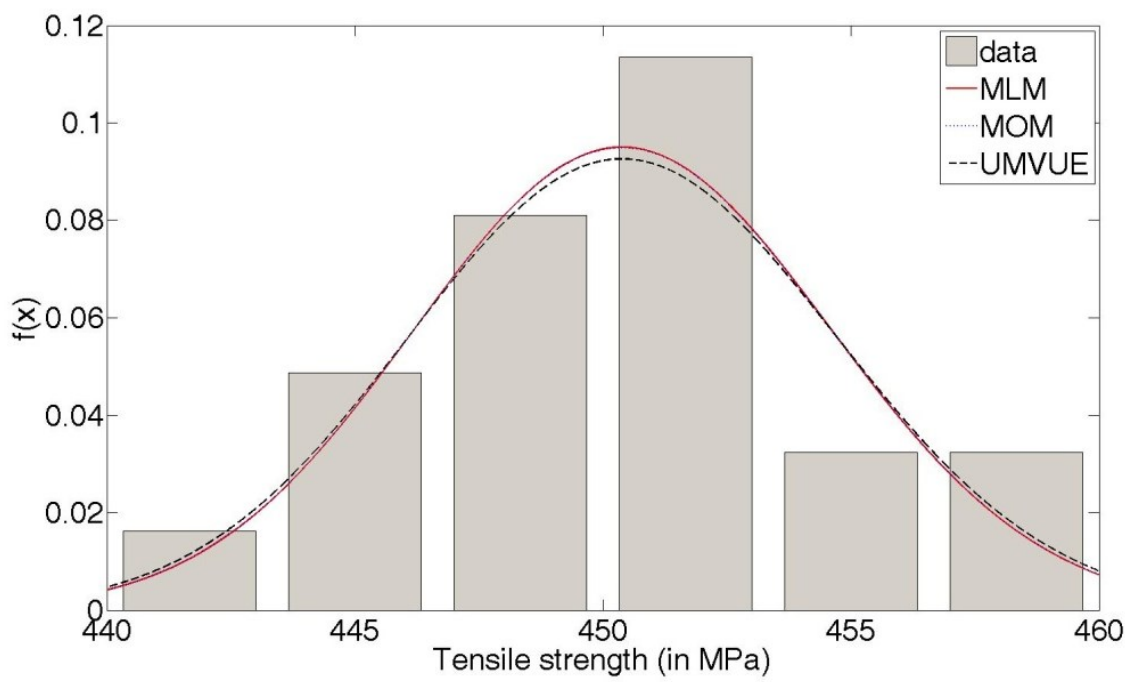

Fig. 2. Fitted PDF and the histogram of the tensile strength

\section{Conclusion}

In the paper, three methods for parameter estimation of lognormal distribution were compared via the Monte Carlo simulation study. To compare the methods, we used bias and root mean square error. According to results, we may conclude that the maximum likelihood method (MLM) and the Finney method (UMVUE) are suitable for estimation of both, $\mu$ and $\sigma^{2}$, parameters of lognormal distribution. To estimate parameter $\mu$, MLM can be preferred. For parameter $\sigma^{2}$ estimation, UMVUE provides better results when $\sigma^{2}$ is small $\left(\sigma^{2} \leq 1\right)$ whereas for larger $\sigma^{2}$, MLM performs better. Furthermore, MLM is computationally less demanding. Method of moments (MOM) performs the worst of all three considered methods.

This work was supported by the Grant 1/0812/17 of the Scientific Grant Agency of the Ministry of Education of the Slovak Republic. 


\section{References}

1. J. F. Ortiz-Yañez, M. R. Piña-Monarrez, Discrimination between the lognormal and Weibull distributions by using multiple linear regression. DYNA, 85(205), 9 - 18, (2018)

2. B. Epstein, The mathematical description of certain breakage mechanism leading to the logarithmic-normal distribution. J Franklin Inst,244, 471-477 (1974)

3. E. Limpert, W. Stahel, M. Abbt, Log-normal Distributions across the Sciences: Keys and Clues. BioScience, 51(5), 341-352 (2001)

4. C. Steele, Use of the lognormal distribution for the coefficients of friction and wear. Reliab Eng Syst Safe, 93(10), 1574 - 1576, (2008)

5. D. J. Finney, On the distribution of a variate whose logarithm is normally distributed. J. R. Stat. Soc. Ser. B. Soc. Ser, 7, 150-161 (1941)

6. L. Jakubovičová, M. Vaško, M. Sága, P. Kopas, Experimental and computational comparative study of the specimens loaded by bending and torsion. MATEC Web Conf., 157(02016), (2018)

7. P. Durčanský, P. Oršanský, Numerical simulation of heat exchanger operation. MATEC Web Conf., 168 (04001), (2018)

8. M. Vaško, M. Handrik, A. Sapietová, J. Handriková, Parallelization of computational algorithms for optimization problems with high time consumption. MATEC Web Conf., 157(02054), (2018)

9. D. Wojtkowiak, I. Malujda, K. Talaśka, Ł. Magdziak, B. Wieczorek, Influence of construction mass distribution on the walking robot's gait stability.Procedia Eng, 177, $419-424,(2017)$

10. A. Vaško, Influence of SiC additive on microstructure and mechanical properties of nodular cast iron. Materials science (Medžiagotyra), 14(4), 311-314, (2008)

11. M. Kukla, J. Górecki, I. Malujda, K. Talaśka, P. Tarkowski, The determination of mechanical properties of magnetorheological elastomers (MREs). Procedia Eng, 177, $324-330,(2017)$

12. H. Akaike, A new look at the statistical model identification, IEEE Trans Automat Contr, 19 (6),716-723, (1974)

13. G. Schwarz, Estimating the dimension of a model, Ann. Statist.,6 (2), 461-464, (1978) 